\title{
Molecular characterization, biofilm analysis and experimental biofouling study of Fusarium isolates from recent cases of fungal keratitis in New York State
}

\author{
Madhu Dyavaiah ${ }^{\dagger 1}$, Rama Ramani ${ }^{\dagger 1,2}$, David S Chu ${ }^{3}$, David C Ritterband ${ }^{4}$, \\ Mahendra K Shah ${ }^{5}$, William A Samsonoff 6 , Sudha Chaturvedi ${ }^{1,2}$ and \\ Vishnu Chaturvedi*1,2
}

Address: ${ }^{1}$ Mycology Laboratory, Wadsworth Center, New York State Department of Health, 120 New Scotland Avenue, Albany, NY 12208-2002, USA, ${ }^{2}$ Department of Biomedical Sciences, School of Public Health, SUNY Albany, 120 New Scotland Avenue, Albany, NY 12208-2002, USA, ${ }^{3}$ Division of Cornea and Refractive Surgery, Institute of Ophthalmology and Visual Science, New Jersey Medical School, Newark, NJ 07101-1709, USA, ${ }^{4}$ Department of Ophthalmology, New York Eye and Ear Infirmary, New York, NY 10003, USA, ${ }^{5}$ Department of Pathology and Laboratory Medicine, New York Eye and Ear Infirmary, New York, NY 10003, USA and ${ }^{6}$ Electron Microscopy Core, Wadsworth Center, New York State Department of Health, Empire State Plaza, Albany, NY 12201-0509, USA

Email: Madhu Dyavaiah - madhud14@yahoo.co.in; Rama Ramani - ramanir@wadsworth.org; David S Chu - chuda@umdnj.edu; David C Ritterband - ritterband@msn.com; Mahendra K Shah - MShah@NYEE.EDU; William A Samsonoff - samsonw@wadsworth.org; Sudha Chaturvedi - schaturv@wadsworth.org; Vishnu Chaturvedi* - vishnu@wadsworth.org

* Corresponding author †Equal contributors

Published: 30 January 2007

BMC Ophthalmology 2007, 7:I doi:10.1/86/147|-24|5-7-I
Received: 2 November 2006

Accepted: 30 January 2007

This article is available from: http://www.biomedcentral.com/I47I-24/5/7/I

(C) 2007 Dyavaiah et al; licensee BioMed Central Ltd.

This is an Open Access article distributed under the terms of the Creative Commons Attribution License (http://creativecommons.org/licenses/by/2.0), which permits unrestricted use, distribution, and reproduction in any medium, provided the original work is properly cited.

\begin{abstract}
Background: To characterize Fusarium isolates from recent cases of fungal keratitis in contact lens wearers, and to investigate fungal association with MoistureLoc solution.

Methods: We studied six fungal isolates from recent cases of keratitis in New York State. The isolates were characterized by nucleotide sequencing and phylogenetic analyses of multiple genes, and then typed using minisatellite and microsatellite probes. Experimental fungal biofilm formation was tested by standard methods. MoistureLoc solutions were tested in biofouling studies for their efficacy in elimination of Fusarium contamination.

Results: Fusarium solani - corneal ulcers ( 2 isolates), lens case ( $I$ isolate), and $F$. oxysporum - corneal ulcer (I isolate), eye ( $I$ isolate), were recovered from five patients. An opened bottle of MoistureLoc solution provided by a patient also yielded $F$. solani. Two distinct genotypes of $F$. solani as well as of $F$. oxysporum were present in the isolated strains. Remarkably, F. solani strains from the lens case and lens solution in one instance were similar, based on phylogenetic analyses and molecular typing. The solution isolate of $F$. solani formed biofilm on contact lenses in control conditions, but not when co-incubated with MoistureLoc solution. Both freshly opened and 3-month old MoistureLoc solutions effectively killed $F$. solani and $F$. oxysporum, when fungal contamination was simulated under recommended lens treatment regimen (4-hr). However, simulation of inappropriate use $(15-60 \mathrm{~min})$ led to the recovery of less than $1 \%$ of original inoculum of $F$. solani or $F$. oxysporum.
\end{abstract}

Conclusion: Temporary survival of $F$. solani and $F$. oxysporum in MoistureLoc suggested that improper lens cleaning regimen could be a possible contributing factor in recent infections. 


\section{Background}

Fungal keratitis due to filamentous fungi is quite common in tropical parts of the world and the southeastern United States [1-3]. Farmers and workers in agro-industries are most at risk for these infections, due to occupation-related corneal abrasions and subsequent fungal infections. In temperate regions, fungal keratitis is most commonly caused by the yeast Candida albicans, although mold keratitis due to Fusarium is being increasingly recognized [46]. Contact lens wearers have an elevated risk for fungal keratitis, but the incidence of Fusarium keratitis is quite rare among this group of patients [7-9]. There has been no report until the beginning of 2006, on the association of multipurpose contact lens solutions with fungal keratitis.

An upsurge in Fusarium keratitis among contact lens wearers was first noticed in 2005 by public health authorities in Singapore. Subsequently, 66 patients were identified from March 2005-February 2006; the characteristics in common among these patients were the use of ReNu with MoistureLoc solution and poor lens hygiene [10]. Two recent reports described a noticeable increase in Fusarium keratitis in contact lens wearers in the US, in the San Francisco Bay Area and in southern Florida [11,12]. A more comprehensive report has documented 176 cases of Fusarium keratitis in 164 patients from various regions in the US [13]. The investigators found significant use of MoistureLoc solution among 45 case patients in their series. Interestingly, they did not recover any Fusarium isolate through extensive sampling of lens solutions from the patients, or from environmental sampling of the plant where the solution was manufactured. Thus, the underlying causes of this perplexing outbreak remain elusive. We investigated recent fungal keratitis cases from New York State including the first case in which Fusarium was isolated from MoistureLoc solution being used by a patient. We also carried out experimental studies with Fusarium isolates in attempts to understand why these infections were associated with the use of MoistureLoc solution.

\section{Methods}

\section{Mycology}

Five Fusarium isolates, recovered from patients' corneal ulcers, contact lenses, or contact lens cases by hospital laboratories in New York and New Jersey, were forwarded to the Mycology Laboratory at the Wadsworth Center (Table 1 ). These isolates were studied in detail for their colony and microscopic characteristics [14,15]. An opened container of MoistureLoc solution being used by a patient for approximately three-months was also submitted for evaluation. An aliquot of this solution was directly plated on Sabouraud's dextrose agar supplemented with antibiotics (chloramphenicol $25 \mu \mathrm{g} / \mathrm{ml}$, gentamicin $40 \mu \mathrm{g} / \mathrm{ml}$ ) for fungal recovery, and the solution was centrifuged at 5000 rpm for $15 \mathrm{~min}$ to recover fungi from the pellet and the supernatant, by plating on culture medium. Additional unopened bottles of MoistureLoc solution were procured from local pharmacies in Albany and New York City before the global recall of this product by the manufacturer. These solutions were processed for fungal testing at the Wadsworth Center and the New York Eye and Ear Infirmary.

\section{Molecular characterization}

Specific identifications and typing of Fusarium isolates were done by PCR and nucleotide sequencing. Fungal DNA extraction involved grinding mycelia in a pestle and mortar under liquid nitrogen, suspending the slurry in lysis buffer (200 mM Tris-HCl, pH 8.5, $250 \mathrm{mM} \mathrm{NaCl}, 25$ mM EDTA, 0.5\%SDS), extraction with phenol: chloroform: isoamyl alcohol, and ethanol precipitation [16]. Fungal ribosomal DNA internal transcribed spacers 1 and 2 (ITS1, ITS2) and nuclear 28S rRNA [17], and Fusariumspecific partial $\beta$-tubulin and elongation factor $(E F-1 \alpha)$ genes were amplified with published primers [18]. Nucleotide sequencing of the PCR amplicons was done on both strands according to the standard methods [19]. Nucleotide sequences were manually edited and compared against the NCBI database and Fusarium database at the Pennsylvania State University [20]. These sequences have been deposited in the GenBank under Accession Numbers

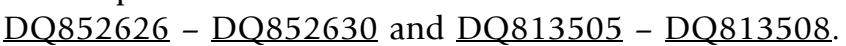
Percentage of nucleotide identity among various genes was compared by ClustalW (v1.4) multiple alignment, using MacVector 7.1.1 software (Accelrys, San Diego, CA). Phylogenetic analyses of nucleotide sequences were done with the PAUP v4 program using a bootstrap method with a Neighbor-Joining or Maximum Parsimony search [21].

Table I: Fusarium species isolated from recent cases of keratitis

\begin{tabular}{lll}
\hline Isolate No. & Source & Identification \\
\hline $23-06$ & Corneal ulcer & F. solani \\
$24-06$ & Corneal ulcer/contact lens & F. solani \\
$158-06$ & Eye & F. oxysporum \\
$159-06$ & Corneal ulcer & F. oxysporum \\
$237-06$ & Lens case & F. solani \\
$238-06$ & MoistureLoc bottle & F. solani
\end{tabular}


Molecular typing of Fusarium isolates was carried out using two random probes: a 15-bp minisatellite probe from M13 bacteriophage [22] and a simple DNA repeat $(\mathrm{GACA})_{4}$ probe [23]. These probes were chosen given that they have been widely used to discriminate between related strains of a variety of pathogenic microorganisms. Three laboratory isolates- F. oxysporum 163-05 (corneal ulcer), F. oxysporum 974-05 (finger nail) and F. solani 1064-05 (corneal ulcer), unrelated to this outbreak, were used as outliers to check robustness of the two genotyping methods. Single M13 (5' GAGGGTGGCGGTTCT 3') and $(\mathrm{GACA})_{4}$ primers were used in PCR reaction. PCR reaction volume of $50 \mu \mathrm{l}$ included $5 \mu \mathrm{l}$ of $10 \times$ PCR buffer with 15 $\mathrm{mM} \mathrm{MgCl}{ }_{2}, 3.0 \mu \mathrm{ldNTP} \operatorname{mix}(10 \mu \mathrm{mol} / \mathrm{L}$ each $), 30 \mathrm{ng}$ primer, 2.5 U Taq DNA polymerase (Perkin Elmer, Foster City, CA, USA). Initial denaturation was at $94^{\circ} \mathrm{C}$ for 20 sec, followed by 35 cycles of denaturation at $94^{\circ} \mathrm{C}$ for 20 sec, annealing at $50^{\circ} \mathrm{C}$ for $1 \mathrm{~min}$, amplification at $72^{\circ} \mathrm{C}$ for $20 \mathrm{sec}$ and final extension $72^{\circ} \mathrm{C}$ for $4 \mathrm{~min}$, in a GenAmp PCR System 2400 (Perkin Elmer). PCR products were concentrated to $20 \mu \mathrm{l}$ and resolved by electrophoresis on $1.4 \%$ agarose gels in Tris-borate-EDTA (TBE) buffer, and were detected by ethidium bromide staining [24].

\section{Biofilm analysis}

Microbial biofilms are an important potential source of infectious propagules in keratitis $[25,26]$. Therefore, the ability of the Fusarium isolates to form biofilms in the laboratory was tested with etafilcon A contact lenses (ACU$\mathrm{VUE}^{\circledR}$, Johnson \& Johnson Vision Care, Inc.) using a published procedure [25]. Briefly, F. solani 238-06 was grown on Sabouraud's dextrose agar slants for 5 days, and the conidia harvested in sterile distilled water. Fungal inoculum comprised of conidial suspension adjusted to 1 $\times 10^{4}$ cells $/ \mathrm{ml}$ with a hemacytometer. A balanced salt solution $\left(0.49 \mathrm{~g} \mathrm{NaCl}, 0.075 \mathrm{~g} \mathrm{KCl}, 0.048 \mathrm{~g} \mathrm{CaCl}_{2}, 0.03 \mathrm{~g}\right.$ $\mathrm{MgCl}_{2}, 0.39 \mathrm{~g}$ sodium acetate $\left\{\mathrm{CH}_{3} \mathrm{COONa}\right\}, 0.17 \mathrm{~g}$ sodium citrate $\{\mathrm{HOC}(\mathrm{COONa})(\mathrm{CH} 2 \mathrm{COONa}) 2\}$, dissolved in $100 \mathrm{ml}$ deionized water, and filter sterilized) was used to promote biofilm formation [25]. Five ml balanced salt solution aliquots were transferred to 25-ml flasks. Half of the flasks received sterile, fresh, ACUVUE contact lens and $10 \mu \mathrm{l}$ of the fungal inoculum. The other flasks received contact lens, fungal inoculum and $1.0 \mathrm{ml}$ MoistureLoc. The negative control was balanced salt solution with contact lens but no fungal inoculum. The flasks were incubated for $48 \mathrm{~h}$ at $25^{\circ} \mathrm{C}$ on a rotator $(180 \mathrm{rpm})$. Some lenses from flasks with balanced salt solution and fungal inoculum but no MoistureLoc were removed at the end of incubation, treated with MoistureLoc for $4 \mathrm{~h}$ and processed for scanning electron microscopy (SEM). The contact lenses were fixed in $2 \%$ glutaraldehyde for $1 \mathrm{~h}$, and dehydrated in 25-100\% ethanol. Dehydrated lenses were critical point dried, coated with gold particles and examined in SEM as described previously [19].

\section{Experimental biofouling}

A previous report indicated that the time elapsed since opening and the storage conditions significantly affected the efficacy of the multipurpose contact lens solutions [27]. We designed a biofouling study based on earlier publications to test for efficacy of MoistureLoc solutions against Fusarium isolates [28,29]. MoistureLoc solutions, purchased from local pharmacies before the global recall of this product, were inoculated with freshly grown Fusarium to test for survival and growth. Both sterile glass test tubes $(20 \times 150 \mathrm{~mm})$ and commercial lens cases (Sight Savers ${ }^{\circledR}$, Bausch \& Lomb, Inc.) were used in these experiments. F. solani 238-06 and F. oxysporum 158-06, from 45 day old slants of Sabouraud's dextrose agar, were suspended in sterile distilled water. The suspension comprised mostly of conidia with rare hyphal fragments. The conidia were counted using a hemacytometer, and adjusted to a final density of $1 \times 10^{4}-10^{7}$ cells $/ \mathrm{ml}$. Ten microliters of these suspensions were used to inoculate $1.0 \mathrm{ml}$ of MoistureLoc. The mix was incubated unstirred at $23^{\circ}-25^{\circ} \mathrm{C}$ for various intervals, and $100 \mu \mathrm{l}$ aliquots were spread on Sabouraud's dextrose agar plates after 15, 30, 60 and $240 \mathrm{~min}$. The positive control included fungal inoculum suspended in sterile water without MoistureLoc. The inoculated plates were incubated at $25^{\circ} \mathrm{C}$ for $48-72 \mathrm{~h}$ and fungal colonies counted and photographed. [28,29]. The biofouling experiments were repeated 5 times.

\section{Results \\ Mycology}

MoistureLoc solution, provided by a patient, was positive for Fusarium species, and quantification of fungal load revealed approximately 50 colonies/ml (figure 1a). Furthermore, the supernatant, and not the pellet from the contaminated solution yielded the fungus (figure 1b). The finding was consistent with moderate recovery of fungal colonies from this solution. Characterization of the morphology and spores of the MoistureLoc isolate (238-06) showed it to most closely resemble F. solani. Mycological investigations of five patient isolates also showed them to be either F. solani or F. oxysporum (Table 1).

\section{Molecular characterization}

Total of five genes including ITS1, ITS2, 28S rRNA, $\beta$ Tubulin, and EF-1 $\alpha$ were used for confirmation of Fusarium species (GenBank Accession Numbers DQ852626 $\underline{\mathrm{DQ} 852630}$ and $\underline{\mathrm{DQ} 813505}$ - DQ813508). Of these genes, sequences from ITS1, ITS2, and EF-1 $\alpha$ possessed enough polymorphism, and therefore, were excellent marker with 99-100\% accuracy for the identification of Fusarium species to be either F. solani or F. oxysporum species complex in the NCBI and Fusarium databases (hereafter referred as F. solani and F. oxysporum). On the contrary, sequences from $28 \mathrm{~S}$ and $\beta$-tubulin genes were highly conserved and therefore, these were only of limited use in fur- 

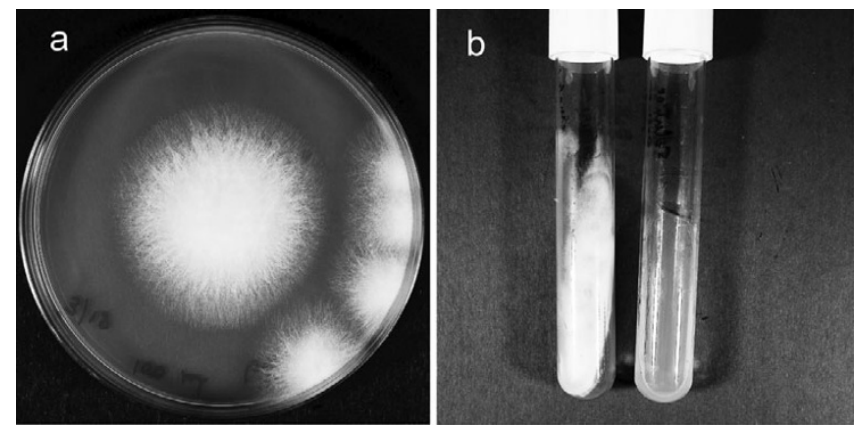

Figure I

Recovery of Fusarium from lens solution. (a) Fusarium solani colonies recovered from $100 \mu \mathrm{l}$ of MoistureLoc cleaning solution provided by a patient (b) F. solani colonies on Sabouraud's dextrose agar slants inoculated with supernatant (left); in contrast no colonies were recovered from the pellet (right) from the same MoistureLoc solution.

ther delineation of Fusarium species. Multiple alignments of EF-1 $\alpha$ sequences of Fusarium species revealed significant polymorphism between $F$. solani and F. oxysporum with $68-73 \%$ identity. Within $F$. solani species, three strains 23-06 (DQ813507), 237-06 (DQ813506), and 238-06 (DQ813505) exhibited 100\% sequence identity and showed $92 \%$ and $84 \%$ identities with strains 1064-05 (DQ852626), and 24-06 (DQ813508), respectively. Similarly, within $F$. oxysporum species, four strains namely 158-05 (DQ852627), 159-06 (DQ852628.), 163-05 (DQ852629), and 974-05 (DQ852630) exhibited small variation in their EF-1 $\alpha$ sequences with 93-95\% identity. Phylogenetic analysis of various gene fragments amplified from these isolates showed distinct clades of $F$. solani and $F$. oxysporum in the test group. Two unrelated strains of $F$. oxysporum (163-05, 974-05) and one F. solani strain (1064-05), used as controls in these studies, also segregated into distinct clades. A representative phylogram from $E F-1 \alpha$ gene fragment is shown in figure 2. Subsequent molecular typing with $\mathrm{M} 13$ and (GACA) ${ }_{4}$ primers generated genotypic patterns that permitted distinct groupings of the test strains (figure 3). Two distinct genotypes of $F$. solani as well as of F. oxysporum were present in the isolated strains. F. solani strain 24-06 genotype was easily separated from the other three strains (23-06, 23706, and 238-06). F. solani from contact lens case (237-06) and from MoistureLoc solution (238-06) showed similar genotypes, by the two probes. In contrast, $F$. oxysporum strains 158-06 and 159-06 yielded two different genotypes. The control F. oxysporum (163-05, 974-05) and F. solani (1064-05) genotypes were distinct.

\section{Experimental studies}

In biofouling studies, F. solani (238-06) formed biofilms on freshly opened contact lenses incubated in balanced

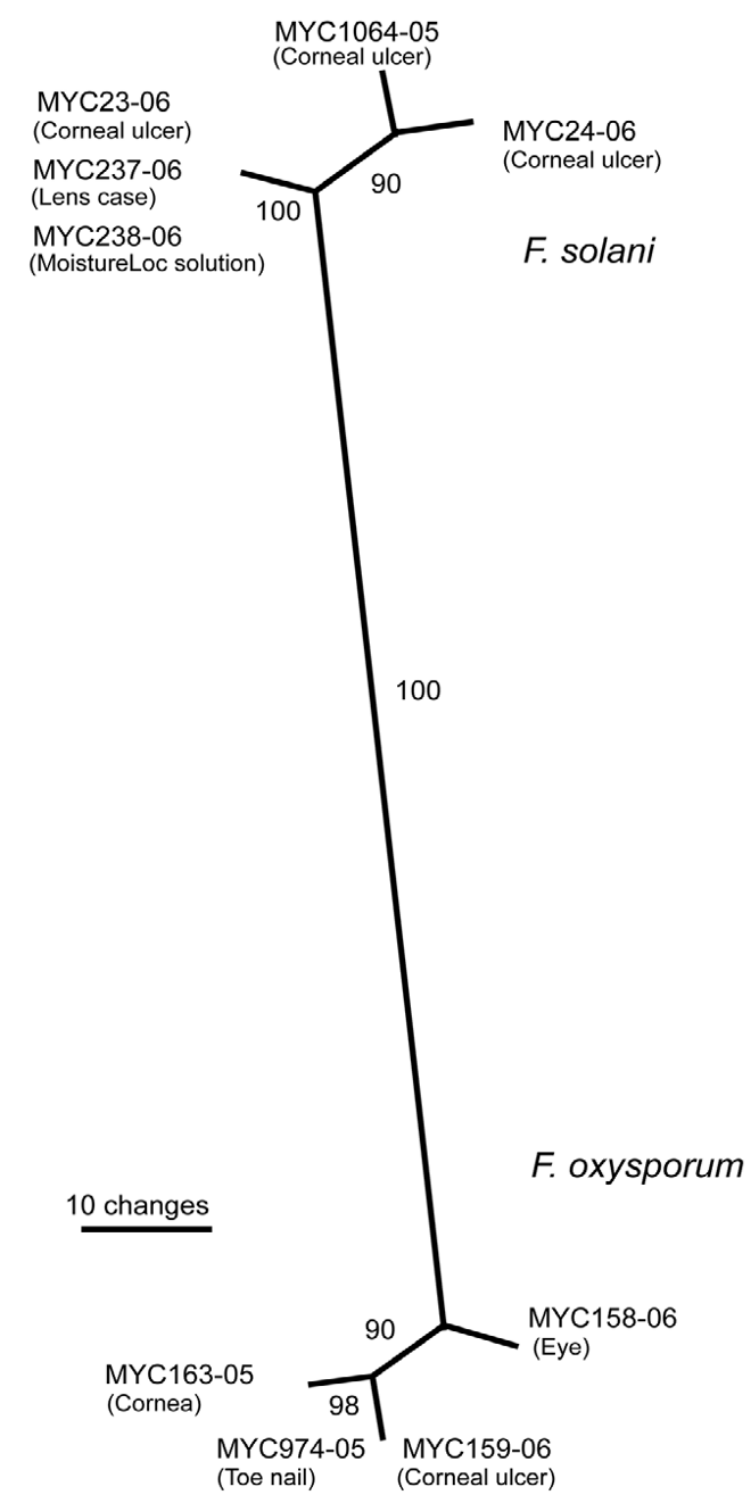

Figure 2

Unrooted parsimony phylogram of Fusarium EF-I $\alpha$ gene sequences. Nucleotide sequences of EF-I $\alpha$ gene were compared by ClustalW multiple alignment using MacVector 7.I.I software (Accelrys, San Diego, CA). Phylogenetic analyses were done with the PAUP v4 program using a bootstrap method with a Neighbor-Joining or Maximum Parsimony search [2I]. The numbers on the branches indicate percent bootstrap values, based on 1000 replicates. Three distinct clades were seen in both the $F$. oxysporum and the $F$. solani strains. The six strains from our investigation came from five different patients: 23-06 (corneal ulcer), 24-06 (contact lens), 158-06 (right eye), 159-06 (corneal ulcer left eye), 237-06 and 238-06 (contact lens case swab and contact lens cleaning solution from the same patient). Three outlier controls were F. oxysporum (163-05) and F. solani (1064-05), previously isolated from unrelated keratitis cases, and F. oxysporum (97405) isolated from finger nail. 

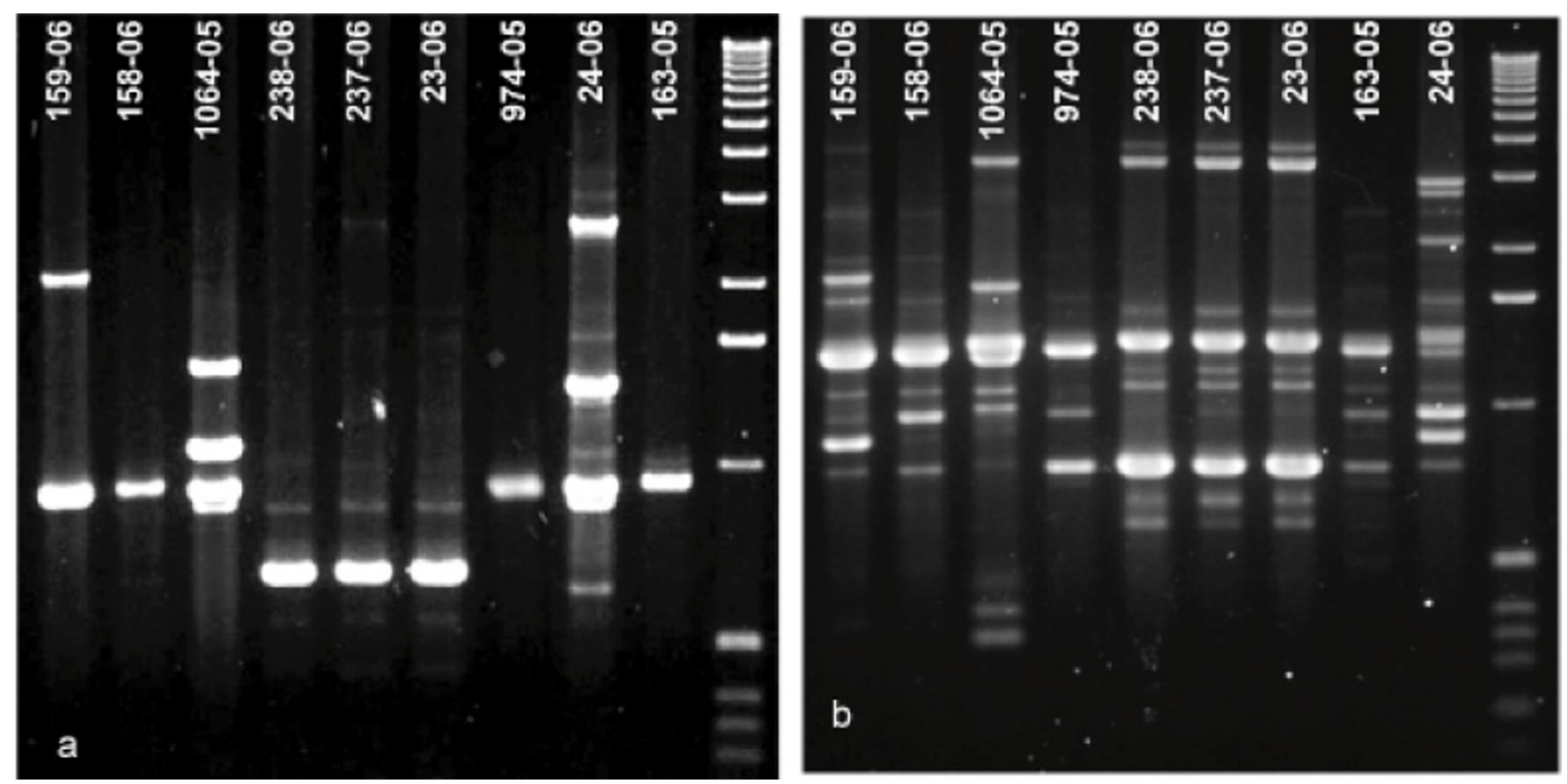

Figure 3

Molecular typing of Fusarium isolates. Molecular typing with (a) minisatellite probe $\{\mathrm{MI} 3\}$, and (b) repeat DNA probe $\{G A C A\}_{4}$ showed distinct genotypes among Fusarium isolates. F. solani strain 24-06 genotype was easily separated from the other three strains $(23-06,237-06$, and 238-06). F. solani from contact lens case (237-06) and from MoistureLoc solution (23806) showed similar genotypes, by the two probes. In contrast, F. oxysporum strains I58-06 and I59-06 yielded two different genotypes. The control F. oxysporum (I63-05, 974-05) and F. solani (I064-05) genotypes were distinct. The lane assignments for 163-05, 974-05, and 24-06 are different in panels (a) and (b).

salt solution (figure 4). Biofilms did not form when contact lenses were incubated in balanced salt solution + MoistureLoc solution. Further, if a biofilm was allowed to form in the salt solution, the treatment with the MoistureLoc sufficed to destroy the biofilm (figure 4c). Similar negative results were obtained when patient isolates were tested for their ability to form biofilms in presence or absence of MoistureLoc (data not shown). Preliminary tests with MoistureLoc suggested that F. solani (238-06) did not survive or grow in shaken cultures kept for 7 days at $30^{\circ} \mathrm{C}$ (data not shown). Different batches of MoistureLoc solutions, procured from local pharmacies before the global recall of this product by the manufacturer, also tested sterile in our hands. MoistureLoc solutions effectively killed F. solani (238-06) and F. oxysporum (158-06) upon experimental inoculation, provided that the recommended length of treatment, 4-hr, was used. Further studies designed to simulate inappropriate cleaning revealed that approximately 25 colonies of $F$. solani $(0.02 \%)$ and 700 colonies of $F$. oxysporum $(0.59 \%)$ could be recovered after $60 \mathrm{~min}$ from the initial 100,000 colony forming units (CFU) used for inoculation of MoistureLoc solution (figures 5, 6). The remaining four test strains also yielded similar survival patterns in these experiments (details not shown). There was no appreciable difference in fungal survival rates, between glass tubes and lens cases used as container for interactions. Similarly, there was no difference in survival pattern between freshly opened MoistureLoc solution and solutions opened 3-months earlier, in these experiments.

\section{Discussion}

Among the significant findings of our study was the recovery of similar F. solani strains from the MoistureLoc solution and from the contact lens case of a patient. To our knowledge, this is the first instance of matched isolations of any Fusarium strain connected with recent cases of fungal keratitis in contact lens wearers [10,13]. However, only one additional isolate of $F$. solani, among the four that we studied, shared this genotype. Also, two F. oxysporum strains in this study showed two distinct genotypes. These observations, although limited suggested that it is unlikely that common or clonal strains were the cause of the infections. This conclusion agrees with the findings of the report about the presence of multiple genotypes of $F$. solani and F. oxysporum in keratitis cases in contact lens wearers in the US [13]. The population make-up of a few F. solani isolates from Singapore and Hong Kong also showed mixed genotypes, albeit with one population predominating; the latter was common in both locales. More 

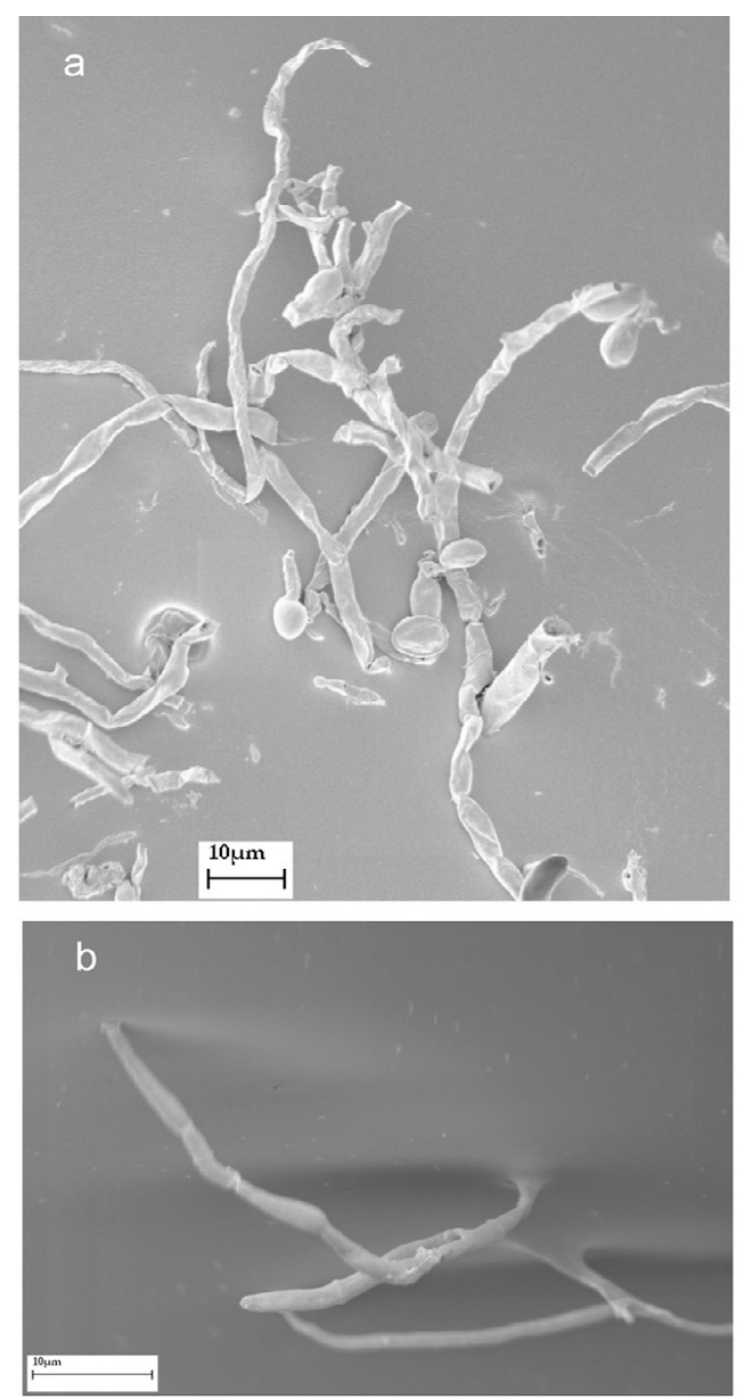

C

\section{2}

Figure 4

Scanning electron micrographs of biofilm formation. (a) F. solani (238-06) incubated with fresh ACUVUE lens and balanced salt solution for $48 \mathrm{~h}$ at $25^{\circ} \mathrm{C}$ on a rotator (I80 rpm). (b) Close-up of the same isolate showing hyphal attachment to the lens surface (c) Disappearance of biofilm when preparation from (a) was treated with MoistureLoc for $4 \mathrm{~h}$.
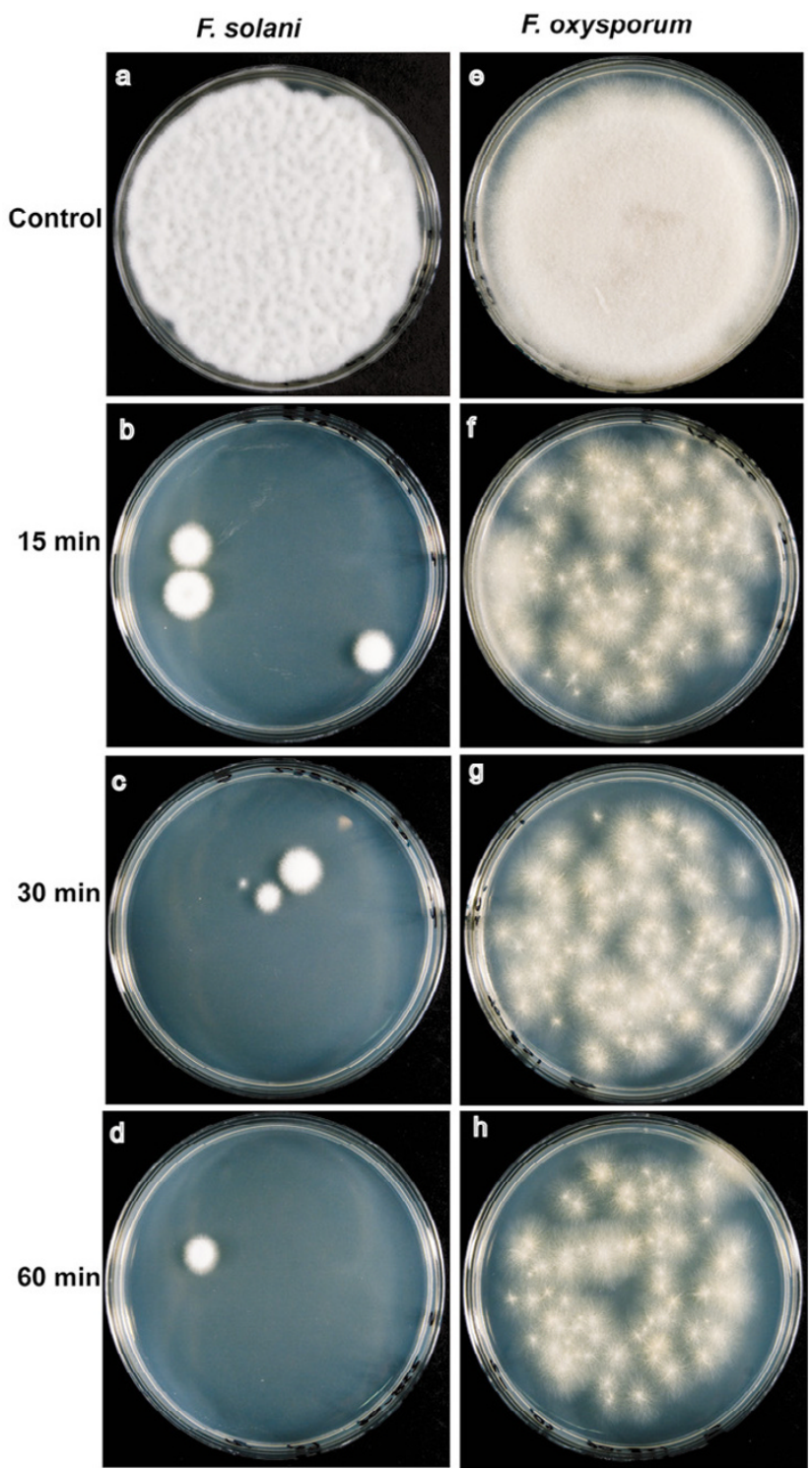

Figure 5

Experimental biofouling studies with F. solani (23806) and $F$. oxysporum (I 58-06). Fungal cells $\left(I \times 10^{5}\right)$ were inoculated into $1.0 \mathrm{ml}$ of MoistureLoc solution, and $100-\mu \mathrm{l}$ aliquots were plated on yeast extract-peptone-dextrose agar at intervals (a-h). Fungal colonies were recovered from MoistureLoc ${ }^{\circledR}$ solutions sampled at I5, 30 and 60 min intervals (b-d) F. solani; (f-h) F. oxysporum. All solutions were sterile after the manufacturer recommended length of treatment, $4 \mathrm{hr}$ (data not shown). The total numbers of colonies recovered are ten-fold of the dilution shown in these illustrations; the data is summarized in figure 6 . Similar results were seen when 3-months old and freshly opened MoistureLoc solutions were compared. The experiments were repeated at least three times; a representative experiment is shown. 


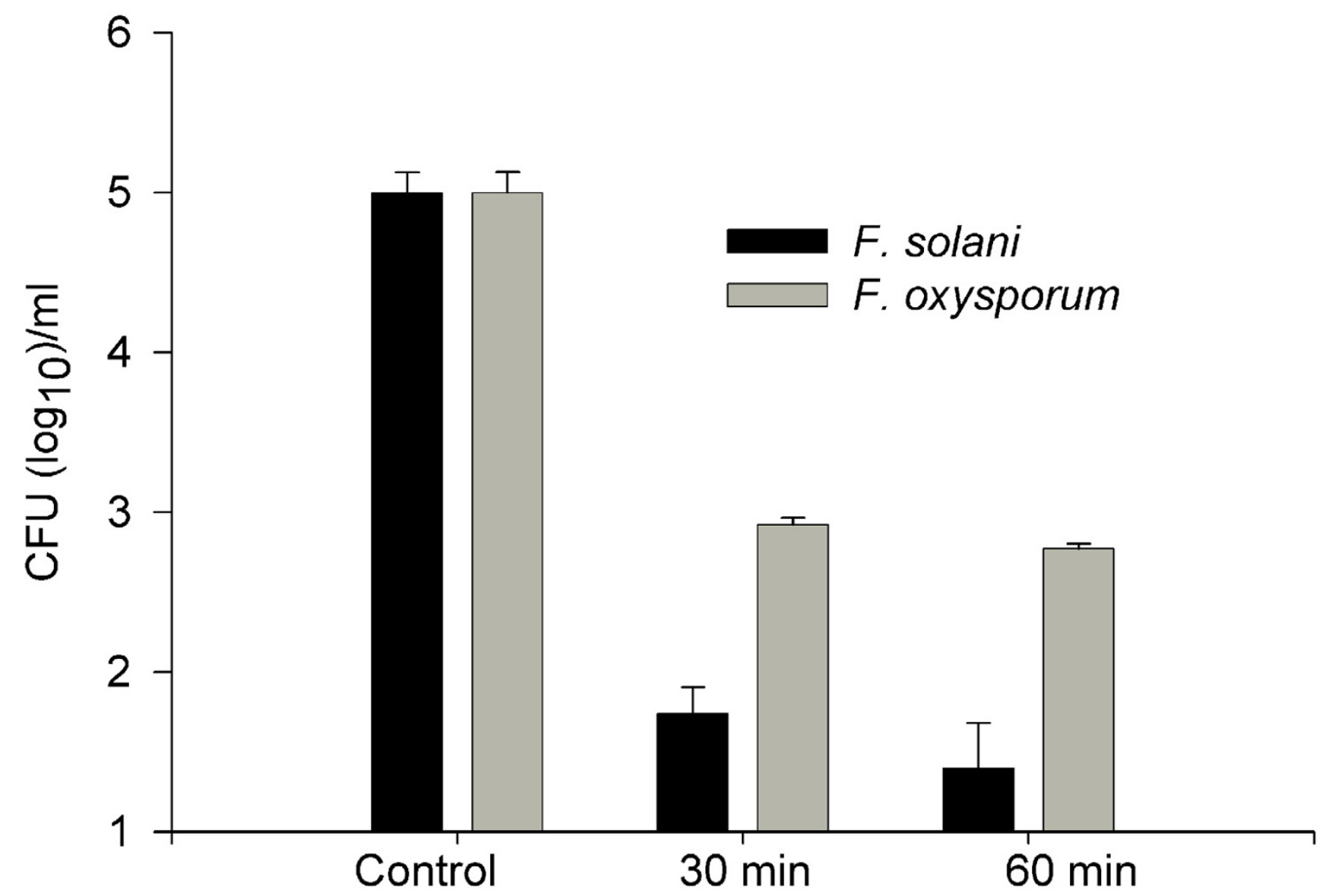

Figure 6

Summary of experimental biofouling studies. The experimental biofouling studies were carried out by introducing $I \times$ $10^{5}$ fungal cells (colony forming units; CFU) of F. solani (238-06) or F. oxysporum (I58-06) into 1.0 ml of MoistureLoc solution. The recovery of CFU after 30 and 60 minutes is shown from three representative experiments (mean \pm SD). The cultures were sterile after recommended $4.0 \mathrm{hrs}$ of incubation (data not shown).

comprehensive studies are indicated to rule out the presence of 'hypervirulent clones' as the cause of recent keratitis cases. It could be pertinent that a number of other investigators have pointed out that clinical strains of Fusarium are generally a mix of genotypes of plant and environmental origin $[13,20,30,31]$.

It was reasonable to suspect that devices such as contact lenses or lens cases are important in the etiology of these infections. In fact, the storage cases are reported to pose an independent risk factor for biofilm formation in mycotic keratitis [26]. We did not receive any relevant specimens for mycology and/or microscopy that would have enabled us to further investigate this aspect. Our limited experimental analyses indicated that both F. solani and F. oxysporum strains were capable of forming biofilms on these devices under permissive conditions, but not in presence of or after recommended treatment with MoistureLoc solution. Based on this evidence, we suggest that the possible sources of Fusarium in keratitis patients are unlikely to include contact lenses or lens cases when MoistureLoc solution is properly used. However, further studies are indicated to examine if contaminated lenses or lens cases could still be important sources of infections independent of lens cleaning regimen.

Our recovery of F. solani from MoistureLoc solution provided by a patient, taken with the previous report of a strong association between MoistureLoc solution and keratitis cases, led us to evaluate the scenarios in which the efficacy of this multipurpose solution would be compromised. We found that MoistureLoc solution completely sterilized $F$. solani and $F$. oxysporum contaminations as long as recommended 4-hr treatment regimen was followed. These observations were consistent with the conclusions of many other reports about the 
overall efficacy of various multipurpose solutions against fungi $[28,29]$. However, it has been documented that the efficacy of cleaning solutions depends on the storage temperature and the time elapsed after opening of the container [27]. Both of these variables were examined to a limited extent in our study; our experiments were carried out with freshly opened containers and with containers opened roughly 3-months earlier with storage at room temperature. Again, no appreciable differences in efficacy were noticed between fresh and aged solutions, experimentally inoculated with $F$. solani and F. oxysporum. The simulation of inappropriate usage of MoistureLoc solution yielded positive growth up to $60 \mathrm{~min}$ with $F$. oxysporum being 30 -fold more resistant than $F$. solani. These results suggested that at least some occurrences of keratitis could result from temporary contamination and/or inadequate cleansing with MoistureLoc solution.

\section{Conclusion}

Temporary survival of F. solani and F. oxysporum in MoistureLoc suggested that improper lens cleaning regimen could be a possible contributing factor in recent infections.

\section{Competing interests}

The author(s) declare that they have no competing interests.

\section{Authors' contributions}

MD: Performed molecular typing and experimental biofouling studies

\section{RR: Performed mycology and biofilm studies}

DSC: Provision of patient material, critical revision of the article

DCR: Provision of patient material, critical revision of the article

MKS: Data collection, provision of materials

WAS: Performed electron microscopy

SC: Data analysis and interpretation, critical revision of the article

VC: Conception and design, analysis and interpretation, writing the draft manuscript, provision of materials and resources

All authors have read and approved the final manuscript.

\section{Acknowledgements}

Josh Schaffzin and Madhu Anand helped with the epidemiology follow ups. Adriana Verschoor provided valuable editorial comments. The Molecular
Genetics Core Facility of the Wadsworth Center performed nucleotide sequencing. We thank the three referees for constructive criticisms and suggestions.

\section{References}

I. Rosa RH Jr., Miller D, Alfonso EC: The changing spectrum of fungal keratitis in south Florida. Ophthalmology 1994, i0I: $1005-1013$.

2. Sharma S: Keratitis. Bioscience Reports 2001, 21:419-444.

3. Thomas PA: Fungal infections of the cornea. Eye 2003, 17:852-862.

4. Tanure MA, Cohen EJ, Sudesh S, Rapuano CJ, Laibson PR: Spectrum of fungal keratitis at Wills Eye Hospital, Philadelphia, Pennsylvania. Cornea 2000, 19:307-3I2.

5. Doczi I, Gyetvai T, Kredics L, Nagy E: Involvement of Fusarium spp. in fungal keratitis. Clin Microbiol Infect 2004, 10:773-776.

6. Ritterband DC, Seedor JA, Shah MK, Koplin RS, McCormick SA: Fungal keratitis at the New York eye and ear infirmary. Cornea 2006, 25:264-267.

7. Wilcox MDP, Holden BA: Contact lens related corneal infections. Bioscience Reports 200I, 2 I:445-46I.

8. Wilhelmus KR, Robinson NM, Font RA, Hamill MB, Jones DB: Fungal keratitis in contact lens wearers. Am J Ophthalmol 1988, I06:708-7|4.

9. Mah-Sadorra JH, Yavuz SG, Najjar DM, Laibson PR, Rapuano CJ, Cohen E): Trends in contact lens-related corneal ulcers. Cornea 2005, 24:5I-58.

10. Khor WB, Aung T, Saw SM, Wong TY, Tambyah PA, Tan AL, Beuerman R, Lim L, Chan WK, Heng W], Lim J, Loh RS, Lee SB, Tan DT: An outbreak of Fusarium keratitis associated with contact lens wear in Singapore. JAMA 2006, 295:2867-2873.

II. Bernal MD, Acharya NR, Lietman TM, Strauss EC, McLeod SD, Hwang DG: Outbreak of Fusarium keratitis in soft contact lens wearers in San Francisco. Arch Ophthalmol 2006, I 24: I05I-I053.

12. Alfonso EC, Cantu-Dibildox J, Munir WM, Miller D, O'Brien TP, Karp CL, Yoo SH, Forster RK, Culbertson WW, Donaldson K, Rodila J, Lee $Y$ : Insurgence of Fusarium keratitis associated with contact lens wear. Arch Ophthalmol 2006, I 24:94I-947.

13. Chang DC, Grant GB, O'Donnell K, Wannemuehler KA, NobleWang J, Rao CY, Jacobson LM, Crowell CS, Sneed RS, Lewis FM, Schaffzin JK, Kainer MA, Genese CA, Alfonso EC, Jones DB, Srinivasan A, Fridkin SK, Park B]: Multistate outbreak of Fusarium keratitis associated with use of a contact lens solution. JAMA 2006, 296:953-963.

14. Nelson PC, Toussoun TA, Marasas WF: Fusarium species: An illustrated manual for identification. University Park, PA, The Pennsylvania State University Press ; 1983.

15. Summerell BA, Salleh B, Leslie JF: A utilitarian approach to Fusarium identification. Plant Disease 2003, 87: I I7- 128.

16. Ausubel FM, Brent R, Kingston RE, Moore DD, Seidman JG, Smith JA, Struhl K: Current protocols in molecular biology. New York, Wiley; 2002.

17. White TJ Bruns, T., Lee, S., Taylor, J.: Amplification and direct sequencing of fungal ribosomal RNA genes for phylogenetics, diagnostics and forensics. In PCR Protocols:A guide to methods and applications Edited by: Innis MAGDHSJJWTJ. San Diego , Academic Press; 1990.

18. O'Donnell K, Cigelnik E, Casper HH: Molecular phylogenetic, morphological, and mycotoxin data support reidentification of the Quorn mycoprotein fungus as Fusarium venenatum. Fungal Genet Biol 1998, 23:57-67.

19. Chaturvedi V, Fan J, Stein B, Behr MJ, Samsonoff WA, Wickes BL, Chaturvedi S: Molecular genetic analyses of mating pheromones reveal intervariety mating or hybridization in Cryptococcus neoformans. Infect Immun 2002, 70:5225-5235.

20. Zhang N, O'Donnell K, Sutton DA, Nalim FA, Summerbell RC, Padhye AA, Geiser DM: Members of the Fusarium solani species complex that cause infections in both humans and plants are common in the environment. J Clin Microbiol 2006, 44:2186-2190.

21. Swafford DL: PAUP*:Phylogenetic analysis using Parsimony and other methods. Sutherland, MA , Sinauer Associates; 2000.

22. Vassart G, Georges M, Monsieur R, Brocas H, Lequarre AS, Christophe $D: A$ sequence in MI3 phage detects hypervariable mini- 
satellites in human and animal DNA. Science 1987, 235:683-684.

23. Ali S, Muller CR, Epplen JT: DNA fingerprinting by oligonucleotide probes specific for simple repeats. Human Genetics 1986, 74:239-243.

24. Chaturvedi S, Dyavaiah M, Larsen RA, Chaturvedi V: Cryptococcus gattii in AIDS Patients, Southern California. Emerg Infect Dis 2005, II:1686-1692.

25. Simmons RB, Buffington JR, Ward M, Wilson LA, Ahearn DG: Morphology and ultrastructure of fungi in extended-wear soft contact lenses. J Clin Microbiol 1986, 24:21-25.

26. McLaughlin-Borlace L, Stapleton F, Matheson M, Dart JK: Bacterial biofilm on contact lenses and lens storage cases in wearers with microbial keratitis. J Appl Microbiol 1998, 84:827-838.

27. Leung $P$, Boost MV, Cho P: Effect of storage temperatures and time on the efficacy of multipurpose solutions for contact lenses. Ophthalmic Physiol Opt 2004, 24:218-224.

28. Miller MJ, Callahan DE, McGrath D, Manchester R, Norton SE: Disinfection efficacy of contact lens care solutions against ocular pathogens. CLAO J 200I, 27:16-22.

29. Rosenthal RA, Henry CL, Stone RP, Schlech BA: Anatomy of a regimen: consideration of multipurpose solutions during noncompliant use. Cont Lens Anterior Eye 2003, 26:17-26.

30. Nelson PE, Dignani MC, Anaissie EJ: Taxonomy, biology, and clinical aspects of Fusarium species. Clin Microbiol Rev 1994, 7:479-504.

31. Healy M, Reece K, Walton D, Huong J, Frye S, Raad, Kontoyiannis DP: Use of the Diversi Lab System for species and strain differentiation of Fusarium species isolates. J Clin Microbiol 2005, 43:5278-5280.

\section{Pre-publication history}

The pre-publication history for this paper can be accessed here:

http://www.biomedcentral.com/1471-2415/7/1/prepub

Publish with Bio Med Central and every scientist can read your work free of charge

"BioMed Central will be the most significant development for disseminating the results of biomedical research in our lifetime. "

Sir Paul Nurse, Cancer Research UK

Your research papers will be:

- available free of charge to the entire biomedical community

- peer reviewed and published immediately upon acceptance

- cited in PubMed and archived on PubMed Central

- yours - you keep the copyright

Submit your manuscript here:

http://www.biomedcentral.com/info/publishing_adv.asp
BioMedcentral 